\title{
Mermelada de arándano y frambuesa: evaluación sensorial, nutricional y de aceptabilidad
}

\author{
Cranberry and raspberry jam: sensory, nutritional \\ and acceptability evaluation
}

\author{
Nelson Eduardo Loyola López* iD Carlos Alberto Acuña Carrasco
}

\section{Acceso Abierto}

*Correspondencia:

nloyola@ucm.cl

Universidad Católica del Maule.

Facultad de Ciencias Agrarias y

Forestales. Escuela de Ciencias

Agrarias. Región del Maule, Chile.

Recibido: 14-03-2021

Aceptado para publicación:

28-07-2021

Publicado en línea: 09-09-2021

Palabras clave:

Aceptabilidad;

ácido ascórbico;

orgánico;

mermelada;

nutricional;

sensorial.

Key words:

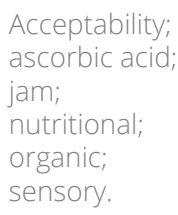

Citación:

Loyola López NE, Acuña Carrasco CA. Mermelada de arándano y frambuesa: evaluación sensorial, nutricional y de aceptabilidad. Magna Scientia UCEVA 2021; 1:118-30. https://doi.org/10.54502/msuceva. v1n1a15.

\section{Resumen}

El objetivo de esta investigación fue obtener una mermelada realizada con mix de arándano (Vaccinium corymbosum L.) y frambuesa (Rubus idaeus L.) orgánicos como materia prima, sellado al vacío y endulzado con azúcar granulada orgánica y/o stevia en polvo orgánica, para su posterior evaluación nutricional, sensorial y de aceptabilidad. Los análisis nutricionales realizados fueron: $i$ ) ácido ascórbico (Vitamina C); ii) azúcares totales; iii) ácido cítrico (acidez); iv) ${ }^{\circ}$ Brix y v) pectato de calcio (pectina). Los análisis sensoriales realizados, se elaboraron a través de una cartilla de evaluación sensorial; sobre la cual, se determinó el sabor, color, textura y aroma; además de realizar la medición de aceptabilidad en conjunto con los panelistas. De acuerdo con los resultados obtenidos en las mermeladas elaboradas, se presentaron los valores promedio mayores en concentración de ácido cítrico $(1.47 \%)$ y pectado de calcio $(0.57 \%)$, respectivamente $\left(\mathrm{T}_{0}\right)$; los valores más altos en referencia a ${ }^{\circ}$ Brix (60.46); azucares totales $(0.8 \%)$; color y textura, los aportó $\mathrm{T}_{1}$; con $\mathrm{T}_{3}$, se obtuvieron los promedios más altos en concentración de ácido ascórbico $(25.7 \mathrm{mg})$, sabor, aroma y aceptabilidad. De este modo, fue factible la realización de mermeladas con base en arándanos y frambuesas orgánicas selladas al vacío y en términos de composición, se logró la afectación de los valores nutricionales, organolépticos y de aceptabilidad.

\section{Abstract}

The aim of this research was to obtain a jam made with a mix of organic blueberry (Vaccinium corymbosum L.) and raspberry (Rubus idaeus L.) as raw material, vacuum sealed and sweetened with organic granulated sugar and/or organic powdered stevia, for subsequent nutritional evaluation, sensory and acceptability. The nutritional analysis performed were as follows: $i$ ) ascorbic acid (Vitamin C); ii) total sugars; iii) citric acid (acidity); iv) ${ }^{\circ}$ Brix and $v$ ) calcium pectate (pectin). The sensory analysis carried out were elaborated through a sensory evaluation card; from which, the flavor, color, texture and aroma, were determined; in addition to carrying out the acceptability measurement in conjunction with the panelists. According to the results obtained in the processed jams, the highest average values were presented in citric acid concentration $(1.47 \%)$ and calcium content $(0.57 \%)$, respectively $\left(\mathrm{T}_{0}\right)$; the highest values in reference to ${ }^{\circ}$ Brix (60.46); total sugars $(0.8 \%)$; color and texture, provided by $\mathrm{T}_{1}$; with $\mathrm{T}_{3}$, the highest averages were obtained in ascorbic acid concentration $(25.7 \mathrm{mg})$, flavor, aroma and acceptability. In this way, it was feasible to make jams based on vacuum-sealed organic blueberries and raspberries, and in terms of composition, it was possible to affect the nutritional, organoleptic and acceptability values. 


\section{Introducción}

El arándano (Vaccinium corymbosum L.) es un cultivo que crece de forma silvestre en el Hemisferio Norte y se encuentra en regiones frías o moderadamente frías. Este fruto, perteneciente al género Vaccinium, incluye más de 450 variedades [1]. Los frutos en la etapa inicial de maduración, se caracterizan por presentar un color blanco, en la siguiente etapa, se tornan de un color rojizo-purpura, para finalmente, llegar a la maduración de la baya de un color azul; esta característica le otorga la denominación de blueberry en inglés [2].

La frambuesa (Rubus idaeus L.), pertenece a la familia de las rosáceas y al género Rubus en donde se incluyen cerca de 2000 variedades [3]. La frambuesa provine del monte Ida, Grecia y su requerimiento climático es templado. El fruto de la frambuesa está compuesto por varias drupas acomodadas en forma de piña, la drupa posee un color rojo escarlata y su piel es aterciopelada; su pulpa es carnosa, jugosa y muy aromática, de un carácter agridulce $[2,4]$.

La confección de mermeladas es regida por la norma del Codex Alimentarius de la Organización de las Naciones Unidas para la Alimentación y la Agricultura (FAO) y la Organización Mundial de la Salud (OMS) [5], para las confituras, jaleas y mermeladas; en el cual, es especificado los requerimientos necesarios para la correcta elaboración de dicho producto. La mermelada es aquella elaborada con fruta entera, pulpa, puré, zumo (jugo), extracto acuoso o cáscara de frutos cítricos, mezclados con azúcares y/o edulcorantes carbohidratos como la miel, con o sin agua y elaborado hasta adquirir una consistencia gelatinosa adecuada, en donde la cantidad de fruta no se debe ser menor al $20 \%$ del cual, no menos del $7.5 \%$ se obtiene del endocarpio, en el producto terminado [5].

La población a nivel mundial, actualmente, presenta una tendencia marcada hacia el consumo de alimentos que sean más sanos, con menores cantidades de químicos que afecten al producto, de fácil preparación y cómodo transporte. Los cambios en los hábitos de alimentación, en conjunto con el incremento del ingreso económico; ha llevado a que la población, consuma productos preferiblemente saludables y sabrosos, además de una fácil preparación y que se encuentren disponibles a lo largo del tiempo [6]. Las nuevas tendencias de alimentación, tienen nuevas exigencias, tales como: $i$ ) el compromiso con el medio ambiente y ii) la disminución y/o la no utilización de productos químicos; lo que se ve reflejado en el incremento de consumidores que buscan productos orgánicos [7]. El fin de este proyecto es satisfacer las nuevas exigencias alimentarias.

La comercialización de productos procesados, ha experimentado un crecimiento significativo durante la última década. A su vez, el consumo de berries se ha incrementado por sus diversas características que aportan valores nutricionales asociados a productos denominados sanos; se proyecta entonces que el consumo de berries, seguirá en aumento [6]. Desde otro punto de vista más económico, Chile, necesita experimentar con productos nuevos, buscar nuevas opciones, que agreguen valor y sustentabilidad a los productos agrícolas; generando de esta forma, nuevas alternativas de comercialización. El objetivo de esta investigación fue obtener una mermelada realizada con mix de arándano ( $V$. corymbosum) y frambuesa (R. idaeus) orgánicos como materia prima, sellado al vacío y endulzado con azúcar granulada orgánica y/o stevia en polvo orgánica, para su posterior evaluación nutricional, sensorial y de aceptabilidad.

\section{Métodos}

\section{Área de estudio}

La investigación fue llevada a cabo en el Laboratorio de la Universidad Católica del Maule, Campus San Isidro, km 6 caminos a Los Niches, Provincia de Curicó-Región del Maule, Chile (Latitud 3501'39.7”S Longitud 71¹1’38.7' Oeste).

\section{Descripción del proceso de elaboración de mermelada}

La descripción del proceso de elaboración de la mermelada con mix de arándano ( $V$. corymbosum) y frambuesa ( $R$. idaeus) y la línea de flujo utilizado, se expone en la figura 1. 


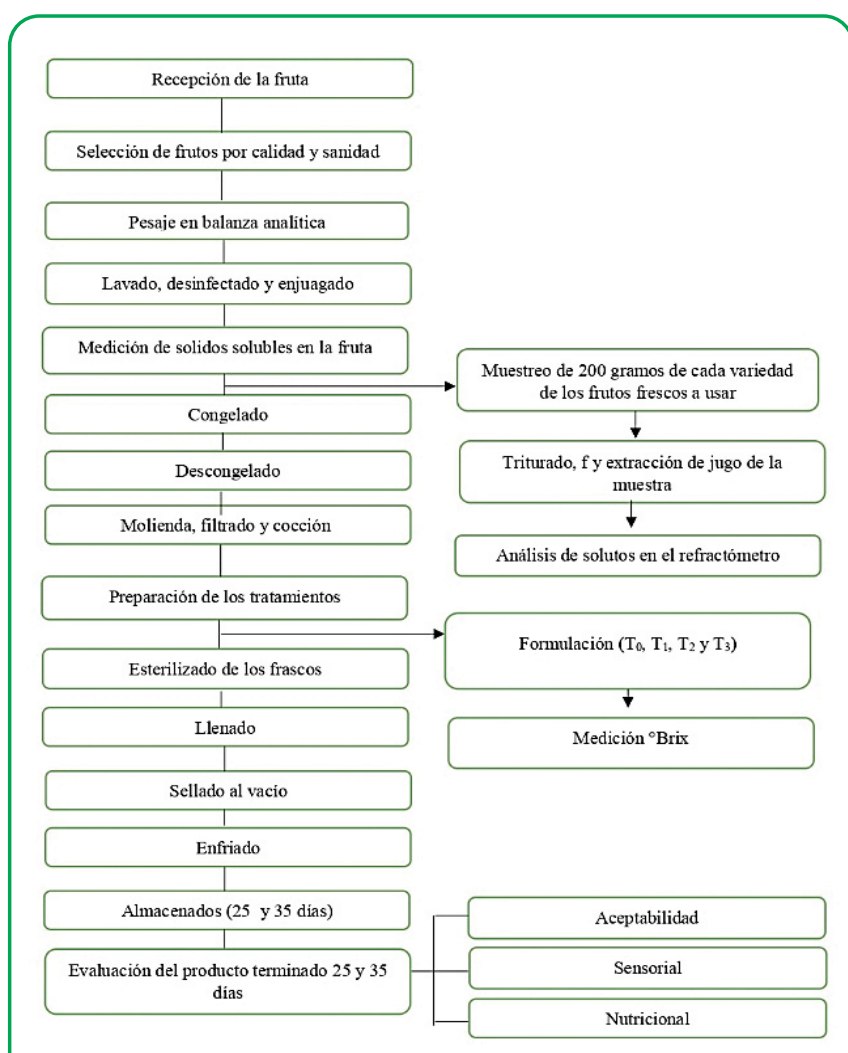

Figura 1. Línea de flujo para la elaboración de mermelada

\section{Recepción de frambuesas y arándanos orgánicos}

Se recibió una cantidad de $18 \mathrm{~kg}$ de arándanos y $12 \mathrm{~kg}$ de frambuesas, obtenidos de la empresa Minuto Verde, ubicada en Parral, Región del Maule, Chile (latitud $36^{\circ}$ 9'8.73" Sur, longitud $71^{\circ} 46^{\prime}, 76^{\prime \prime}$ Oeste), con el objeto de ser procesados. En las cajas correspondientes a una temperatura ambiente; los productos fueron transportados hasta la región de Los Niches, para ser sometidos al proceso de congelamiento.

\section{Selección de frutos por criterios de sanidad}

Una vez seleccionados los frutos por criterios de calidad y sanidad (figura 1), se descartaron aquellas frutas que no cumplían con los estándares y condiciones adecuadas; principalmente, frutas que exhibían la presencia de una enfermedad o ataque de microorganismos patógenos relacionados con enfermedades postcosecha; con el fin de garantizar la inocuidad del producto. Las frutas seleccionadas (figura 1), se pesaron en una balanza analítica (Mettler Toledo ${ }^{\mathrm{TM}}$, modelo AB204-s) [8].

\section{Lavado y esterilización de la materia prima}

El lavado y esterilización de la materia prima se realizó con una solución de $3 \mathrm{~L}$ de hipoclorito de sodio $(\mathrm{NaClO})$, el cual contenía cloro $\left(\mathrm{Cl}_{2}\right)$ a una concentración de 50 ppm para disminuir la carga bacteriana al mínimo, sin afectar la calidad del producto. Los frutos fueron posteriormente sumergidos y agitados cuidadosamente en la solución durante aproximadamente 1.5 minutos. Acto seguido, se lavaron los frutos con agua potable para eliminar cualquier tipo de residuo; posteriormente, fueron sometidos a proceso de congelamiento en una nevera.

\section{Medición de sólidos solubles ( $\left.{ }^{\circ} \mathrm{Brix}\right)$}

Se determinaron los sólidos solubles de los frutos de arándano y frambuesa, utilizando una muestra de $200 \mathrm{~g}$. Luego, se efectuaron las lecturas de ${ }^{\circ}$ Brix en el refractómetro (Atago ${ }^{\mathrm{TM}}$, modelo atc-1), haciendo uso de la norma AOAC (Official Methods of Analysis) $932.12[9]$.

\section{Molienda y filtrado}

Después del descongelado de la fruta, se procedió a triturar la fruta en una juguera hasta lograr una pasta uniforme; la cual, fue filtrada con un género para extraer las semillas de los frutos.

\section{Preparación del medio de cobertura}

El medio de cobertura, correspondió a distintos tratamientos: $\mathrm{T}_{0}, \mathrm{~T}_{1}, \mathrm{~T}_{2}$ y $\mathrm{T}_{3}$, respectivamente. Para cada tratamiento, se utilizaron distintos aditivos, como sigue: i) $\mathrm{T}_{0}=$ sin aditivo; $i$ i) $\mathrm{T}_{1}=$ con azúcar; $\left.i i i\right) \mathrm{T}_{2}=$ con stevia y iv) $\mathrm{T}_{3}=$ azúcar + stevia. La mermelada se preparó a una temperatura de cocción de $98^{\circ} \mathrm{C}$, determinada por termómetro, siguiendo los protocolos establecidos [10].

\section{Formulación de las mermeladas}

En las mermeladas elaboradas, se realizaron cuatro formulaciones distintas, las que fueron denominas como tratamientos (tabla 1); en las cuales, varía la cantidad de fruta utilizada y principalmente, los aditivos que poseen. La cantidad de materia prima en conjunto con los aditivos y conservantes, es denominada dosis. La tabla 1, expone los distintos tratamientos en conjunto con las dosis utilizadas. 
Tabla 1. Formulación de las mermeladas elaboradas

\begin{tabular}{|c|c|}
\hline Tratamiento & Dósis \\
\hline $\mathrm{T}_{0}$ & $\begin{array}{l}50 \% \text { de fruta de arándano y un } 50 \% \text { de fruta de } \\
\text { frambuesa }+1.5 \% \text { de conservante }[80 \% \text { Benzoato } \\
\text { de Sodio }\left(\mathrm{C}_{7} \mathrm{H}_{5} \mathrm{NaO}_{2)} \text { y } 20 \% \text { Sorbato de Potasio }\right. \\
\left(\mathrm{C}_{6} \mathrm{H}_{7} \mathrm{KO}_{2}\right]\end{array}$ \\
\hline $\mathrm{T}_{1}$ & $\begin{array}{l}25 \% \text { de fruta de arándano y } 25 \% \text { de fruta de } \\
\text { frambuesa }+50 \% \text { azúcar }+1.5 \% \text { de conservante } \\
{\left[80 \% \text { Benzoato de Sodio }\left(\mathrm{C}_{7} \mathrm{H}_{5} \mathrm{NaO}_{2}\right) \text { y } 20 \%\right.} \\
\text { Sorbato de Potasio }\left(\mathrm{C}_{6} \mathrm{H}_{7} \mathrm{KO}_{2}\right]\end{array}$ \\
\hline $\mathrm{T}_{2}$ & $\begin{array}{l}40 \% \text { de fruta de arándano y } 40 \% \text { de fruta de frambuesa } \\
+20 \% \text { manzana Granny Smith orgánica } 1.5 \% \text { de } \\
\text { conservante }\left[80 \% \text { Benzoato de Sodio }\left(\mathrm{C}_{7} \mathrm{H}_{5} \mathrm{NaO}_{2}\right)\right. \\
\text { y } 20 \% \text { Sorbato de Potasio }\left(\mathrm{C}_{6} \mathrm{H}_{7} \mathrm{KO}_{2}\right]+\text { Stevia } \\
\text { (equivalente al poder edulcorante del azúcar } 50 \%)\end{array}$ \\
\hline $\mathrm{T}_{3}$ & $\begin{array}{l}32.5 \% \text { de fruta de arándano y } 32.5 \% \text { de fruta } \\
\text { de frambuesa }+10 \% \text { manzana Granny Smith } \\
\text { orgánica }+25 \% \text { de azúcar }+ \text { Stevia (equivalente } \\
\text { al poder edulcorante del azúcar } 25 \%)+1.5 \% \text { de } \\
\text { conservante }\left[80 \% \text { Benzoato de Sodio }\left(\mathrm{C}_{7} \mathrm{H}_{5} \mathrm{NaO}_{2}\right)\right. \\
\text { y } 20 \% \text { Sorbato de Potasio }\left(\mathrm{C}_{6} \mathrm{H}_{7} \mathrm{KO}_{2}\right]\end{array}$ \\
\hline
\end{tabular}

\section{Formulación balance de sólidos solubles}

Esta fórmula es diseñada con el fin de evaluar la cantidad de solidos solubles necesarios para cumplir con la cantidad de sólidos solubles establecida por el Codex Alimentarius [5], la cual debe estar entre 60 - 65\% en el producto terminado. La tabla 2, enseña la formulación con base en $1 \mathrm{~kg}$ de fruta.

Tabla 2. Balance solidos solubles

\begin{tabular}{cccc}
\hline $\begin{array}{c}\text { Materia } \\
\text { prima }\end{array}$ & G & ${ }^{\circ}$ Brix & g sólidos solubles \\
\hline Fruta & 1000 & $\begin{array}{c}{ }^{\circ} \text { Brix de la } \\
\text { fruta }(\%)\end{array}$ & $\begin{array}{c}\% \text { sólidos solubles de } \\
\text { la fruta }\end{array}$ \\
Azúcar & $\begin{array}{c}(1000-\text { sólidos } \\
\text { solubles de la } \\
\text { fruta })\end{array}$ & $1(100 \%)$ & $\begin{array}{c}(1000-\text { sólidos } \\
\text { solubles de la fruta })\end{array}$ \\
\hline
\end{tabular}

Los sólidos solubles expuestos en la tabla 2, obedecen a la siguiente determinación:

$$
\text { Sólidos solubles }=\frac{\sum g \text { sólidos solubles }}{\sum g} \times 100
$$

Adicionalmente, se realizó la formulación del rendimiento para obtener la concentración necesaria para la formulación de mermeladas.

$$
\text { Rendimiento }=\frac{\sum g \times \% \text { sólidos solubles }}{\%^{\circ} \text { Brix norma }(65 \diamond)}
$$

Con base en lo anterior, se logró conocer cuál es la cantidad de agua que se debió evaporar para lograr la concentración de sólidos solubles adecuada. Para la problemática del uso de Stevia como edulcorante, no rige la norma Codex Alimentarius [5].

\section{Frascos}

Se utilizaron frascos de vidrio de $250 \mathrm{ml}$, los cuáles fueron desinfectados y esterilizados con una solución desinfectante de $200 \mathrm{ppm}$ de hipoclorito de sodio ( $\mathrm{NaClO}$ ). La capacidad de los envases, se consideró de acuerdo con semejanzas en el mercado actual; el cual, posee $240 \mathrm{ml}$ de mermelada correspondiente a cada tratamiento.

\section{Sellado al vacío}

El sellado al vacío se realizó por medio de temperatura, para evitar la presencia de oxígeno; el cual, se ejecutó a temperaturas entre $\operatorname{los} 55^{\circ}-98^{\circ} \mathrm{C}$, durante 45 segundos [11].

\section{Parámetros a evaluar}

\section{Sólidos solubles}

Determinación de solidos según Método Oficial AOAC 932.12 sólidos solubles en frutas y productos frutales; método refractométrico [9]. Se evaluaron los sólidos solubles de los tratamientos $\left(\mathrm{T}_{0}, \mathrm{~T}_{1}, \mathrm{~T}_{2}\right.$ y $\left.\mathrm{T}_{3}\right)$ tres veces por muestra a partir del día 25 y 35 de almacenado.

\section{Determinación de ácido ascórbico (vitamina C) en mermeladas}

Se determinó la cantidad de ácido ascórbico (Vitamina C) a través del método AOAC 967.21 [12], con mediciones a los 25 y 35 días después de la elaboración de la mermelada.

\section{Determinación de azúcares totales}

La determinación de azucares totales se determinó siguiendo los lineamientos expuestos por la norma AOAC 923.02 [13] con mediciones a los 25 y 35 días después de la elaboración de la mermelada. 


\section{Determinación de acidez}

Mediante la norma AOAC 942.15 [14], se determinó la acidez que poseía la mermelada.

\section{Determinación de pectato de calcio (Pectina)}

Se determinó la cantidad de pectina mediante el método gravimétrico de Carré y Haynes, descrito por Kirk et al. [15].

\section{Evaluación sensorial y aceptabilidad}

La evaluación sensorial se realizó posterior a 25 y 35 días de almacenamiento de las mermeladas, en los cuales, se comprobarán que no representan ningún riesgo para la salud humana. Este proceso se realizó de forma aleatoria, aplicando cartillas de evaluación sensorial y de aceptabilidad, para 15 panelistas.

\section{Diseño experimental}

Eldiseñoestadístico aplicadofue bloques completamente al azar (BCA) e incluyó la evaluación a partir del análisis de varianza. En los casos que se encontraron diferencias significativas, se sometieron a la prueba de honestidad de Tukey con un nivel de significancia del 0.05. Los datos fueron analizados con el programa estadístico STATGRAPHICS ${ }^{\circledR}$ Centurión versión 16, 2009.

\section{Resultados}

Análisis de propiedades nutricionales y parámetros químicos

\section{Análisis de ${ }^{\circ}$ Brix}

Las mermeladas elaboradas fueron evaluadas y los resultados obtenidos, se promediaron como se demuestra en la tabla 3 .

Tabla 3. Valores promedio de ${ }^{\circ}$ Brix en 25 y 35 días

\begin{tabular}{ccc}
\hline Tratamiento & Prom. ${ }^{\circ}$ Brix 25 días & Prom. ${ }^{\circ}$ Brix 35 días \\
\hline $\mathrm{T}_{1}$ & $60.40^{\mathrm{a}}$ & $60.46^{\mathrm{a}}$ \\
$\mathrm{T}_{3}$ & $39.96^{\mathrm{b}}$ & $40.10^{\mathrm{b}}$ \\
$\mathrm{T}_{2}$ & $12.43^{\mathrm{c}}$ & $12.70^{\mathrm{c}}$ \\
$\mathrm{T}_{0}$ & $12.73^{\mathrm{c}}$ & $12.86^{\mathrm{c}}$ \\
\hline
\end{tabular}

Promedios con la misma letra no difieren significativamente entre sí ( $p>0.05$ ANOVA seguido de la prueba Tukey).
Las mermeladas elaboradas con los tratamientos $\mathrm{T}_{0}$ (fruta orgánica sin edulcorante) y $\mathrm{T}_{2}$ (fruta orgánica + Stevia), no presentaron diferencias significativas entre ellos (figura 2 ) respecto a los ${ }^{\circ}$ Brix medidos.

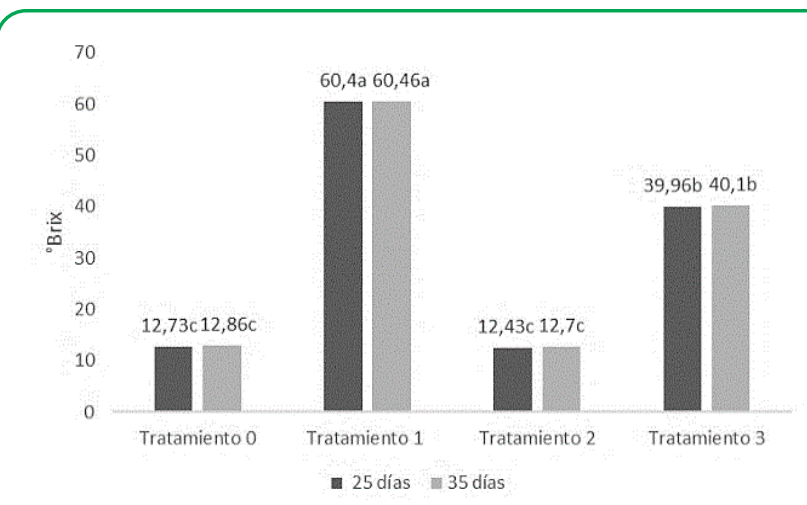

Figura 2. Valores promedio de ${ }^{\circ}$ Brix en 25 y 35 días

Los valores promedio que no presentan una letra en común, son significativamente diferentes según test de Tukey $(\mathrm{p}<=0.05)$

La adición de azúcar granulada orgánica en las mermeladas elaboradas con el tratamiento $\mathrm{T}_{1}$, permitió un incremento significativo en la concentración de los sólidos solubles (valores $\mathrm{T}_{1} \mathrm{y}_{2}$ ), debido a que la fruta en un comienzo, presentó entre 11 y $12^{\circ}$ Brix.

\section{Análisis del contenido de ácido cítrico}

La cantidad de ácido cítrico que se extrajo de cada mermelada elaborada, fue expresada en porcentaje de acuerdo con una muestra de $2 \mathrm{~g}$ (tabla 4).

Tabla 4. Valores promedio de ácido cítrico en 25 y 35 días

\begin{tabular}{ccc}
\hline Tratamiento & Ácido cítrico 25 días & Ácido cítrico 30 días \\
\hline $\mathrm{T}_{0}$ & $1.46 \% \%_{\mathrm{a}}$ & $1.47 \%_{\mathrm{a}}$ \\
$\mathrm{T}_{2}$ & $1.18 \%_{\mathrm{b}}$ & $1.12 \%_{\mathrm{b}}$ \\
$\mathrm{T}_{3}$ & $0.92 \%_{\mathrm{c}}$ & $0.90 \%_{\mathrm{c}}$ \\
$\mathrm{T}_{1}$ & $0.81 \%_{\mathrm{d}}$ & $0.79 \%_{\mathrm{d}}$ \\
\hline
\end{tabular}

Promedios con la misma letra no difieren significativamente entre sí ( $p>0.05$ ANOVA seguido de la prueba Tukey).

La mermelada elaborada que presentó una mayor concentración de ácido cítrico fue obtenida con el tratamiento $\mathrm{T}_{0}$, obteniéndose promedios entre $1.46 \% \mathrm{y}$ $1.47 \%$ de ácido cítrico; el tratamiento que presentó una 
menor concentración de ácido cítrico fue el tratamiento $\mathrm{T}_{1}$ con promedios de concentración entre $0.81 \%$ y $0.79 \%$. Los resultados se exponen en la figura 3 .

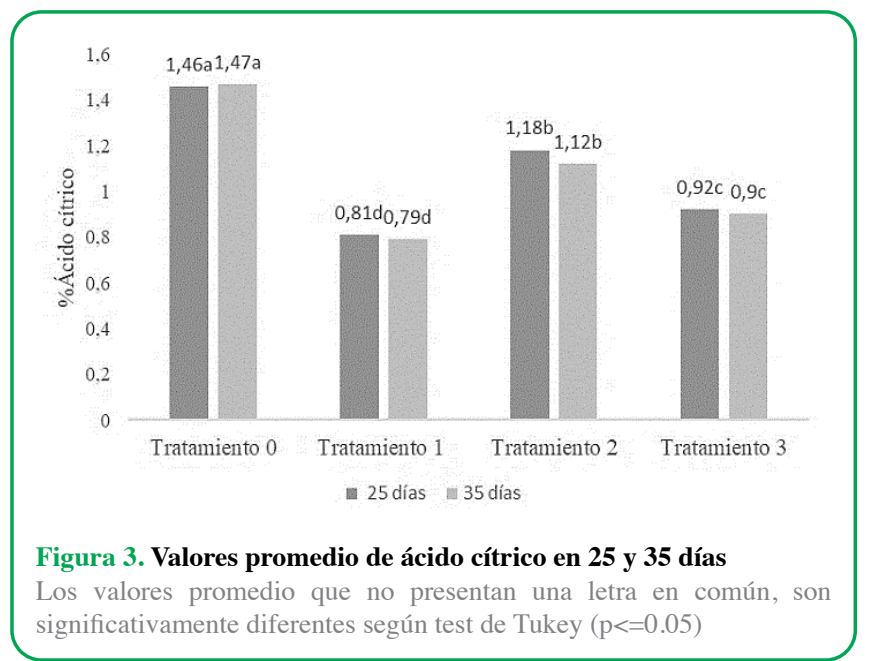

En las mermeladas elaboradas, respecto al porcentaje de ácido cítrico, todos los tratamientos presentaron diferencias significativas entre ellos, debido a la concentración de fruta que poseían (figura 3).

\section{Análisis del contenido de ácido ascórbico (vitamina C)}

En las mermeladas elaboradas, se realizó la medición de ácido ascórbico en 100gr de muestra. La tabla 5, muestra la cantidad promedio en mg de ácido ascórbico evaluada.

Tabla 5. Valores promedio en mg de ácido ascórbico en 25 y 35 días

\begin{tabular}{ccc}
\hline Tratamiento & $\begin{array}{c}\text { Prom. ácido } \\
\text { ascórbico 25 días }\end{array}$ & $\begin{array}{c}\text { Prom. ácido } \\
\text { ascórbico 35 días }\end{array}$ \\
\hline $\mathrm{T}_{3}$ & $24.50_{\mathrm{a}}$ & $25.70_{\mathrm{a}}$ \\
$\mathrm{T}_{2}$ & $22.20_{\mathrm{b}}$ & $23.56_{\mathrm{b}}$ \\
$\mathrm{T}_{1}$ & $20.00_{\mathrm{c}}$ & $19.80_{\mathrm{c}}$ \\
$\mathrm{T}_{0}$ & $14.40_{\mathrm{d}}$ & $16.40_{\mathrm{d}}$ \\
\hline
\end{tabular}

Promedios con la misma letra no difieren significativamente entre sí ( $p>0.05$ ANOVA seguido de la prueba Tukey).

El análisis estadístico reflejó diferencias significativas en todos los tratamientos de las mermeladas realizadas, respecto a la cantidad de ácido ascórbico. La mermelada elaborada con el tratamiento $\mathrm{T}_{3}$, fue la que presentó la mayor concentración de vitamina $\mathrm{C}$, en donde los arándanos y frambuesas fueron mezclados con azúcar orgánica, stevia y manzana orgánica; obteniendo $24.5 \mathrm{mg}$ de ácido ascórbico. $100 \mathrm{~g}^{-1}$ de muestra. El segundo tratamiento que presentó una mayor concentración de ácido ascórbico fue el $\mathrm{T}_{2}$, el cual, contiene como adición, manzana y Stevia; obteniendo $20.2 \mathrm{mg}$ de ácido ascórbico. $100 \mathrm{~g}^{-1}$ de muestra (figura 4).

\section{Análisis del contenido de azúcares totales}

Los resultados obtenidos de las mermeladas elaboradas, son expresados en porcentaje de acuerdo a las muestras realizadas de $100 \mathrm{ml}$ de mermelada (tabla 6 ).

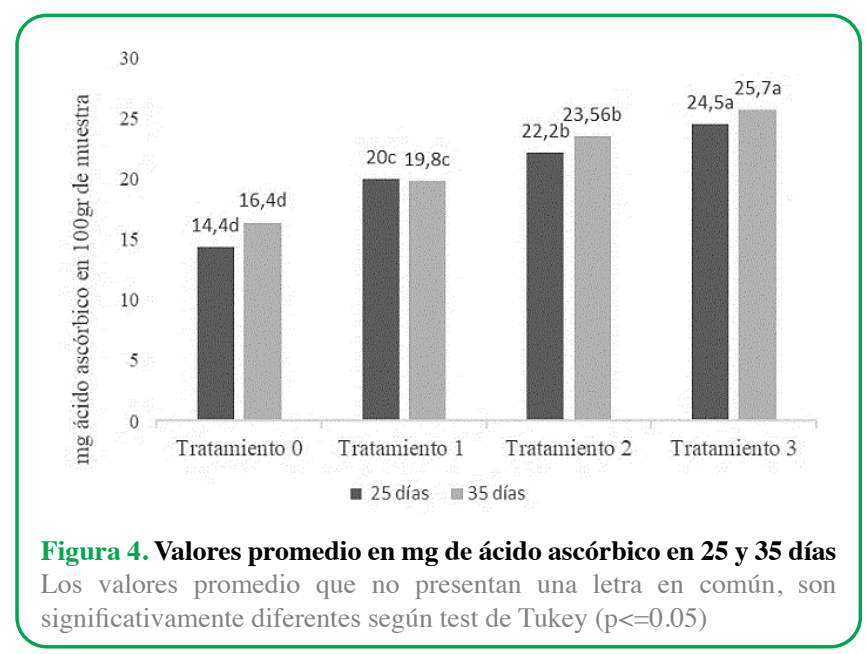

Tabla 6. Valores promedios de azúcares totales en 25 y 35 días

\begin{tabular}{ccc}
\hline Tratamiento & $\begin{array}{c}\text { Prom. Azúcares } \\
\text { totales 25 días }\end{array}$ & $\begin{array}{c}\text { Prom. Azúcares } \\
\text { totales 35 días }\end{array}$ \\
\hline $\mathrm{T}_{1}$ & $0.80 \%{ }_{\mathrm{a}}$ & $0.78 \%_{\mathrm{a}}$ \\
$\mathrm{T}_{3}$ & $0.70 \%_{\mathrm{b}}$ & $0.70 \%_{\mathrm{b}}$ \\
$\mathrm{T}_{0}$ & $0.18 \%_{\mathrm{c}}$ & $0.19 \%_{\mathrm{c}}$ \\
$\mathrm{T}_{2}$ & $0.19 \%_{\mathrm{d}}$ & $0.20 \%_{\mathrm{d}}$ \\
\hline
\end{tabular}

Promedios con la misma letra no difieren significativamente entre sí ( $p>0.05$ ANOVA seguido de la prueba Tukey).

La mermelada elaborada con el tratamiento $\mathrm{T}_{1}$, fue la que presentó la mayor concentración de azúcares totales, debido a que este tratamiento, estaba compuesto por azúcar y fruta orgánica. La cantidad de azúcares totales obtenidos en la mermelada elaborada con 
el tratamiento $\mathrm{T}_{1}$, fluctúo entre $0.8 \%$ y $0.78 \%$. Las mermeladas elaboradas con los tratamientos $\mathrm{T}_{2}$ y $\mathrm{T}_{0}$, no presentaron diferencias significativas entre ellos en cuanto al contenido de azúcares totales (figura 5).

\section{Análisis del contenido de pectato de calcio (Pectina)}

Los resultados obtenidos de las mermeladas elaboradas, fueron promediados y expresados en porcentaje (tabla 7).

La mermelada con el tratamiento que presentó la mayor cantidad de pectato de calcio, fue el $\mathrm{T}_{0}$, elaborado únicamente con fruta orgánica y conservantes; los promedios obtenidos con el tratamiento $\mathrm{T}_{0}$, se encontraban entre $0.55 \%$ y $0.57 \%$ (tabla 7 ).

Tabla 7. Valores promedio de pectato de calcio en 25 y 35 días

\begin{tabular}{ccc}
\hline Tratamiento & Pectina 25 días & Pectina 30 días \\
\hline $\mathrm{T}_{0}$ & $0.55 \%_{\mathrm{a}}$ & $0.57 \%_{\mathrm{a}}$ \\
$\mathrm{T}_{2}$ & $0.48 \% \%_{\mathrm{b}}$ & $0.46 \%{ }_{\mathrm{b}}$ \\
$\mathrm{T}_{1}$ & $0.37 \%_{\mathrm{c}}$ & $0.39 \%_{\mathrm{c}}$ \\
$\mathrm{T}_{3}$ & $0.39 \%_{\mathrm{c}}$ & $0.40 \%_{\mathrm{c}}$ \\
\hline
\end{tabular}

Promedios con la misma letra no difieren significativamente entre sî́ ( $p>0.05$ ANOVA seguido de la prueba Tukey).

La segunda mermelada que obtuvo una mayor cantidad de pectato de calcio, fue la elaborada con el tratamiento $\mathrm{T}_{2}$, la cual presentó un tango entre $0.48 \%$ y $0.46 \%$ de pectato de calcio (figura 5).

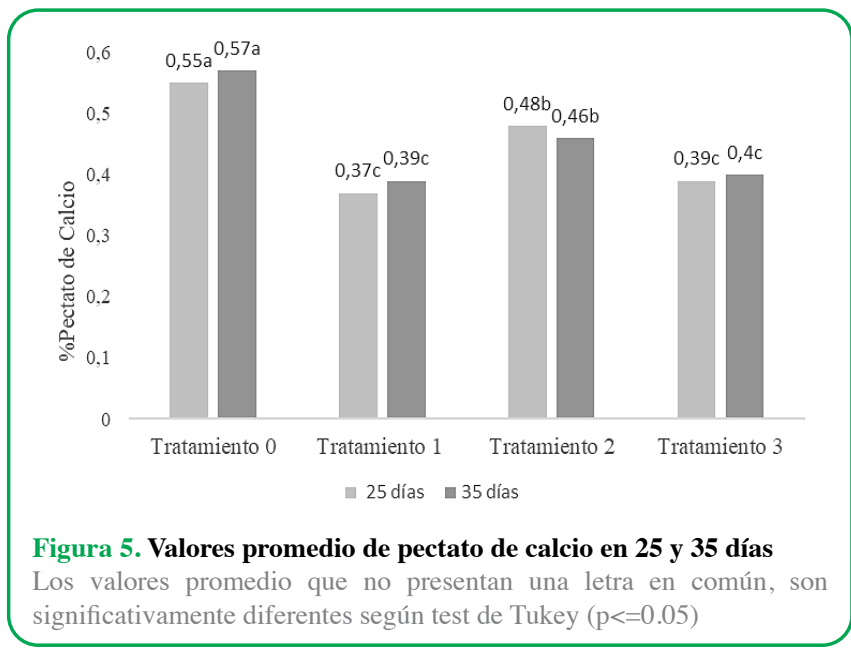

\section{Evaluación sensorial de las mermeladas}

\section{Análisis sensorial: Sabor}

En las mermeladas realizadas, los valores obtenidos oscilaron entre 0 y 13 , donde 13 , fue el valor máximo "dulce" (tabla 8).

La mermelada elaborada con el tratamiento $\mathrm{T}_{0}$, fue la que presentó un sabor más "insípido" percibido por los panelistas; los degustadores evaluaron el tratamiento entre los valores 3.33 y 2.83 y lo catalogaron como "cercano a insípido" (tabla 8).

Tabla 8. Valores promedio de sabor en 25 y 35 días

\begin{tabular}{ccc}
\hline Tratamiento & Sabor 25 días & Sabor 30 días \\
\hline $\mathrm{T}_{3}$ & $12.07_{\mathrm{a}}$ & $12.23_{\mathrm{a}}$ \\
& $($ dulce) & (dulce) \\
$\mathrm{T}_{1}$ & $10.13_{\mathrm{b}}$ & $10.50_{\mathrm{b}}$ \\
& (cercano a dulce) & (cercano a dulce) \\
$\mathrm{T}_{2}$ & $7.27_{\mathrm{c}}$ & $7.60_{\mathrm{c}}$ \\
& (no muy dulce) & (no muy dulce) \\
$\mathrm{T}_{0}$ & $3.33_{\mathrm{d}}$ & $2.83_{\mathrm{d}}$ \\
& (cercano a insípido) & (cercano a insípido) \\
\hline
\end{tabular}

Promedios con la misma letra no difieren significativamente entre sí ( $p>0.05$ ANOVA seguido de la prueba Tukey).

La mermelada elaborada con el tratamiento $\mathrm{T}_{3}$, fue la que presentó el sabor "más dulce", donde los panelistas evaluaron la mermelada con Stevia, azúcar orgánica y manzana orgánica; obteniendo valores entre $12.07 \mathrm{y}$ 12.23 , considerada finalmente como "dulce" (figura 6).

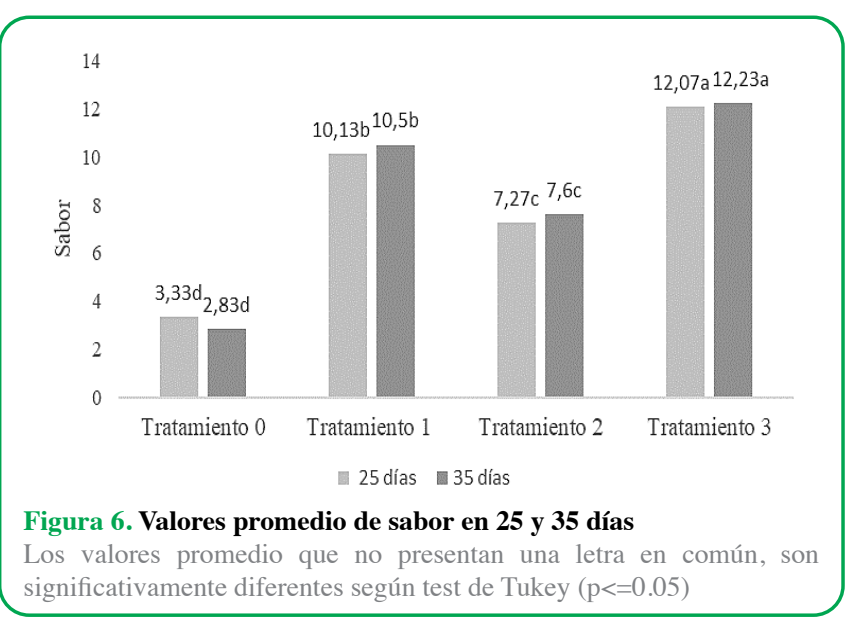


De acuerdo con el análisis estadístico realizado a las mermeladas elaboradas, todos los tratamientos evaluados, presentaron diferencias significativas entre ellos; respecto al atributo sensorial del sabor, por los distintos componentes edulcorantes en la mermelada elaborada (figura 6).

\section{Análisis sensorial: Color}

Los valores obtenidos oscilaron entre 0 ("color claro") y 13 ("color oscuro") (tabla 9).

Tabla 9. Valores promedio de color en 25 y 35 días

\begin{tabular}{|c|c|c|}
\hline Tratamiento & Color 25 días & Color 30 días \\
\hline \multirow[t]{2}{*}{$\mathrm{T}_{1}$} & $11.93_{\mathrm{a}}$ & $12.03_{\mathrm{a}}$ \\
\hline & (oscuro) & (oscuro) \\
\hline \multirow[t]{2}{*}{$\mathrm{T}_{3}$} & $9.03_{\mathrm{b}}$ & $9.50_{b}$ \\
\hline & $\begin{array}{l}\text { (medianamente } \\
\text { oscuro) }\end{array}$ & $\begin{array}{l}\text { (medianamente } \\
\text { oscuro) }\end{array}$ \\
\hline $\mathrm{T}_{2}$ & $5.00_{c}$ & $4.80_{c}$ \\
\hline \multirow[t]{2}{*}{$\mathrm{T}_{0}$} & $\begin{array}{c}\text { (levemente oscuro) } \\
5.10_{c}\end{array}$ & $\begin{array}{c}\text { (levemente oscuro) } \\
5.50_{c}\end{array}$ \\
\hline & (levemente oscuro) & (levemente oscuro) \\
\hline
\end{tabular}

Promedios con la misma letra no difieren significativamente entre sí ( $p>0.05$ ANOVA seguido de la prueba Tukey).

Los panelistas percibieron que las mermeladas elaboradas con el tratamiento $\mathrm{T}_{1}$, presentaron un color oscuro, el cual, contenía azúcar y fruta orgánica con valores promedios resultantes entre 11.93 y 12.03 , que lo catalogaban como "oscuro" (tabla 9). De la misma manera, los panelistas evaluaron con "levemente oscuro" a las mermeladas elaboradas con los tratamientos $\mathrm{T}_{0} \mathrm{y} \mathrm{T}_{2}$, respectivamente, los cuales no contenían adición de azúcar; resultando entre 4.8 y 5.5 , caracterizado finalmente como "levemente oscuro" (tabla 9; figura 7).

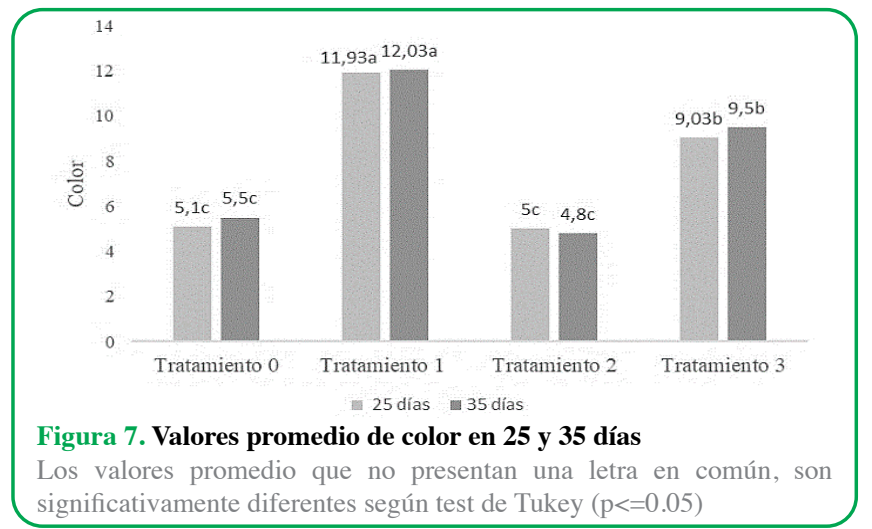

\section{Análisis sensorial: Textura}

De acuerdo con la cartilla de evaluación sensorial realizada a las mermeladas elaboradas, en donde el valor 0 es el mínimo y 13 el máximo; los resultados fueron promediados como se demuestra en la tabla 10 .

Tabla 10. Valores promedio de textura en 25 y 35 días

\begin{tabular}{ccc}
\hline Tratamiento & Textura 25 días & Textura 30 días \\
\hline $\mathrm{T}_{1}$ & $12.07_{\mathrm{a}}$ & $11.96_{\mathrm{a}}$ \\
& (firme) & (firme) \\
$\mathrm{T}_{3}$ & $10.07_{\mathrm{b}}$ & $10.22_{\mathrm{b}}$ \\
& (medianamente & (medianamente firme) \\
& firme) & $8.74_{\mathrm{c}}$ \\
$\mathrm{T}_{2}$ & $8.73_{\mathrm{c}}$ & (levemente firme) \\
& (levemente firme) & $2.17_{\mathrm{d}}$ \\
$\mathrm{T}_{0}$ & $2.10_{\mathrm{d}}$ & (suave) \\
\hline
\end{tabular}

Promedios con la misma letra no difieren significativamente entre sí ( $p>0.05$ ANOVA seguido de la prueba Tukey).

De acuerdo con la percepción de los panelistas, las mermeladaselaboradas con el tratamiento $\mathrm{T}_{0}$, presentaron una menor textura, obteniendo resultados entre $2.1 \mathrm{y}$ 2.17, caracterizado como "suave". Las mermeladas elaboradas con el tratamiento $\mathrm{T}_{1}$, presentaron los mayores valores en cuanto a textura percibido por los panelistas; el que consiste en la mezcla de fruta orgánica y azúcar orgánica, otorgando la calificación de 12.07, catalogada como "firme" (tabla 10; figura 8).

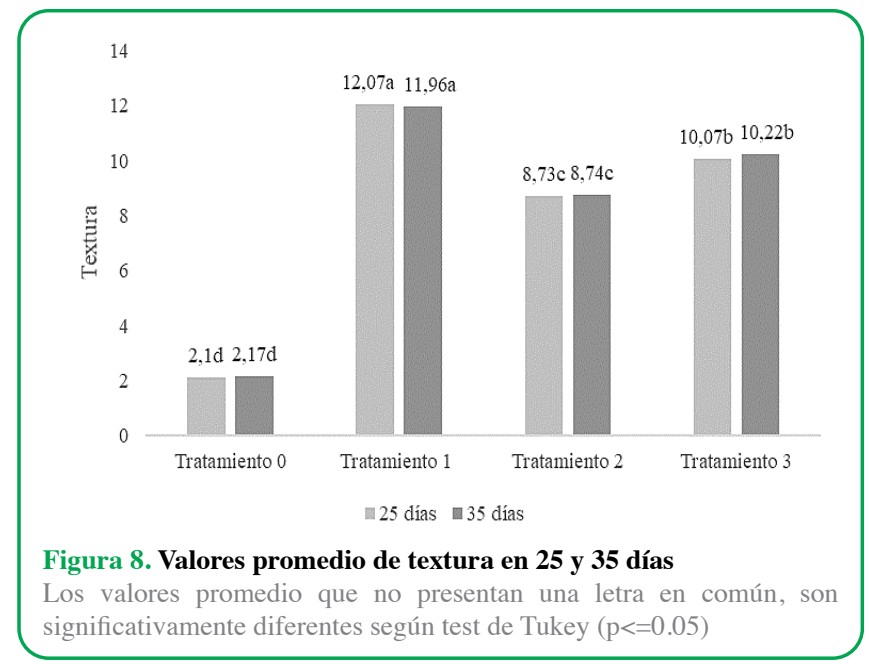




\section{Análisis sensorial: Aroma}

Mediante el uso de cartillas sensoriales, se evaluó el atributo aroma, percibido por los panelistas en las mermeladas elaboradas. En una escala de 0-13 en donde 0, indica un aroma insípido y 13, un aroma frutal (tabla 11).

Tabla 11. Valores promedio de aroma en 25 y 35 días

\begin{tabular}{ccc}
\hline Tratamiento & Aroma 25 días & Aroma 30 días \\
\hline $\mathrm{T}_{3}$ & $10.80_{\mathrm{a}}$ & $10.83_{\mathrm{a}}$ \\
& (aroma frutal) & (aroma frutal) \\
$\mathrm{T}_{1}$ & $8.6_{\mathrm{b}}$ & $8.67_{\mathrm{b}}$ \\
& (medianamente & (medianamente frutal) \\
& frutal) & $6.87_{\mathrm{c}}$ \\
$\mathrm{T}_{2}$ & $6.50_{\mathrm{c}}$ & (levemente frutal) \\
& (levemente frutal) & $4.50_{\mathrm{d}}$ \\
$\mathrm{T}_{0}$ & $4.33_{\mathrm{d}}$ & (cercano a insípido) \\
& (cercano a insípido)
\end{tabular}

Promedios con la misma letra no difieren significativamente entre sí ( $p>0.05$ ANOVA seguido de la prueba Tukey).

La mermelada que presentó un mayor aroma ("frutal") según los panelistas, fue el tratamiento $\mathrm{T}_{3}$, con valores entre 10.8 y 10.83, catalogado como "aroma frutal", en los cuales, los panelistas percibieron e indicaron que posee un aroma "agradable y frutal". El tratamiento que presentó un aroma "más insípido" fue el $\mathrm{T}_{0}$, con valores entre 4.33 y 4.5 "cercano a insípido", en el cual, los panelistas lo señalaron como aroma "acido". El tratamiento $\mathrm{T}_{1}$, presentó valores entre 8.6 y 8.67 , catalogado como "medianamente frutal", e incluso, un aroma a "caramelo", señalado por los degustadores (tabla 11; figura 9).

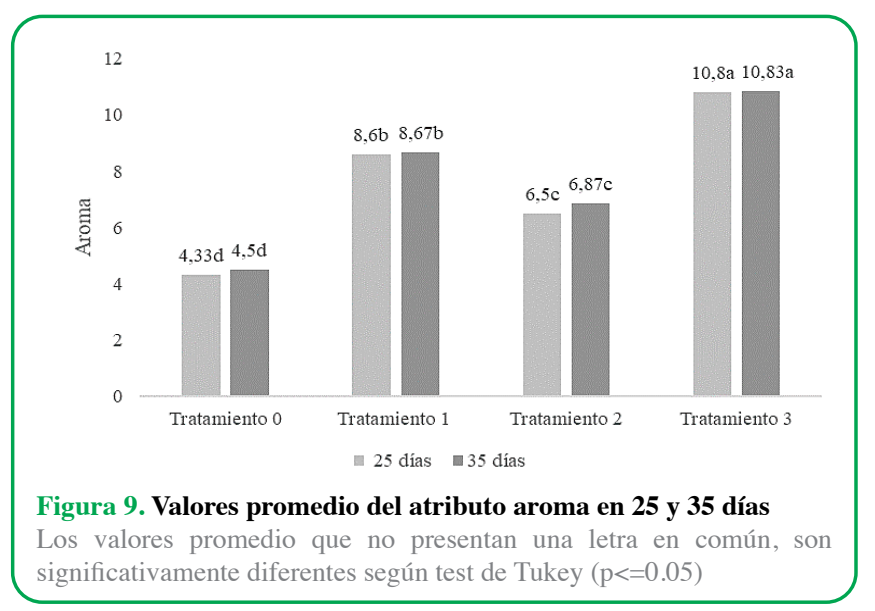

\section{Análisis de aceptabilidad}

Los valores obtenidos de las mermeladas elaboradas, oscilaron entre $0-9$, en donde 0 , fue el valor mínimo ("me disgusta extremadamente") y 9 , el valor máximo ("me gusta extremadamente") (tabla 12).

Tabla 12. Valores promedio de aceptabilidad en 25 y 35 días

\begin{tabular}{ccc}
\hline Tratamiento & Aceptabilidad 25 días & Aceptabilidad 30 días \\
\hline $\mathrm{T}_{3}$ & $7.8_{\mathrm{a}}$ & $8.0_{\mathrm{a}}$ \\
& (me gusta mucho) & (me gusta mucho) \\
$\mathrm{T}_{1}$ & $7.0_{\mathrm{b}}$ & $7.2_{\mathrm{b}}$ \\
& (me gusta & (me gusta \\
& moderadamente) & moderadamente) \\
$\mathrm{T}_{2}$ & $6.2_{\mathrm{c}}$ & $6.0_{\mathrm{c}}$ \\
& (me gusta levemente) & (me gusta levemente) \\
$\mathrm{T}_{0}$ & $1.6_{\mathrm{d}}$ & $1.8_{\mathrm{d}}$ \\
& (me disgusta & (me disgusta \\
& extremadamente) & extremadamente) \\
\hline
\end{tabular}

Promedios con la misma letra no difieren significativamente entre sí ( $p>0.05$ ANOVA seguido de la prueba Tukey).

Los panelistas aceptaron de mejor forma aquellas mermeladas elaboradas con el tratamiento $\mathrm{T}_{3}$ (tabla 12); de acuerdo con los panelistas, fue clasificado con un promedio de 8 "me gusta mucho", lo que indica que, el producto genera satisfacción en el consumidor; por lo que indicaron que la mermelada resultó "agradable y de buen sabor". La mermelada que presentó una menor aceptabilidad fue la elaborada con el tratamiento $\mathrm{T}_{0}$, con valores entre 1.6 y 1.8 "me disgusta extremadamente", en donde los panelistas comentaron que la mermelada $\mathrm{T}_{0}$ era de un "sabor demasiado ácido y mala" (tabla 12; figura 10).

\section{Discusión}

Después de 25 y 35 días de la elaboración de las mermeladas, la medición de ${ }^{\circ}$ Brix en la mermelada elaborada con el tratamiento $\mathrm{T}_{1}$ (fruta orgánica + azúcar), se acoge a la norma establecida por el Codex Alimentarius [5], el cual se rige en medida de las mermeladas que poseen azúcar en forma de aditivo; producto que debe contener al menos un $60-65 \%{ }^{\circ}$ Brix en su presentación final. Se menciona en el Codex 
Alimentarius [5], que se exceptúa de esta norma a las mermeladas que contengan edulcorantes como la stevia.

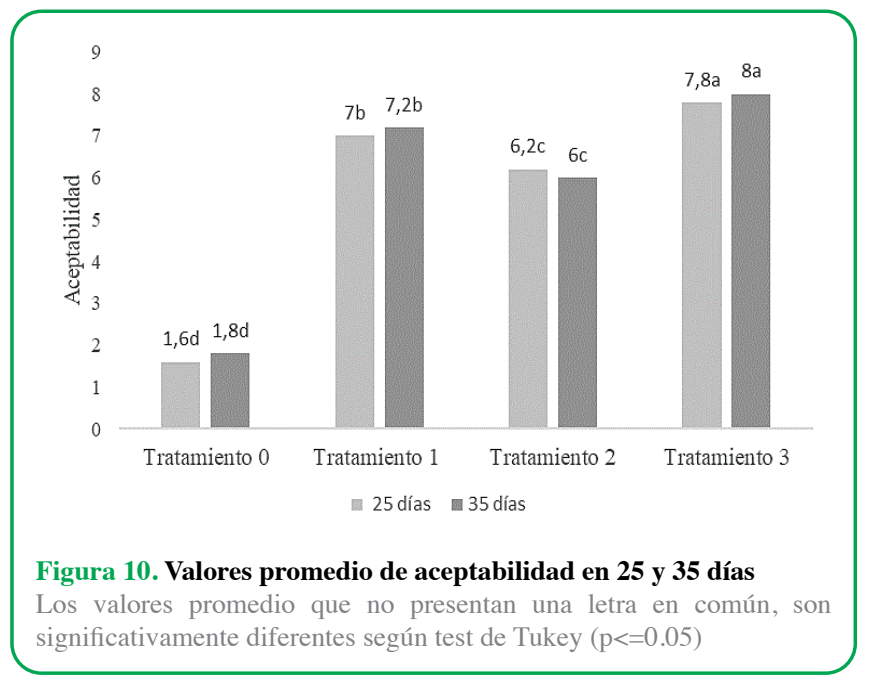

La mermelada elaborada con el tratamiento $\mathrm{T}_{1}$, presentó los mayores valores de ${ }^{\circ}$ Brix, con un total de 60.4 debido a que contenía azúcar orgánica, arándanos y frambuesas. Este efecto se produce según la FAO [16], dado que la adición de azúcar a las mermeladas, permitió el aumento de $\operatorname{los}^{\circ} \mathrm{Brix}$, lo que fue corroborado por el tratamiento $\mathrm{T}_{3}$ (fruta orgánica + stevia + azúcar + manzana), tratamiento que presentó la menor cantidad de ${ }^{\circ}$ Brix, al poseer $50 \%$ menos de azúcar granulada respecto al tratamiento $T_{1}$.

El proceso de elaboración de la mermelada permite incrementar los ${ }^{\circ}$ Brix por el efecto de la evaporación del agua, lo que favoreció una mayor concentración de los sólidos solubles [16]. La mermelada elaborada con el tratamiento $\mathrm{T}_{0}$ fue la que presentó la mayor cantidad de ácido cítrico, debido a que estaba compuesta únicamente de fruta y sin edulcorante. El tratamiento $\mathrm{T}_{2}$ presentó una menor cantidad de ácido cítrico dado que contenía $20 \%$ de manzana, la cual posee menos ácido cítrico que los arándanos y la frambuesa [17]. Los tratamientos $\mathrm{T}_{1}$ y $\mathrm{T}_{3}$, disminuyeron la cantidad de ácido cítrico debido a que posee una menor concentración de fruta.

La adición de un componente frutal extra en este caso, el uso de manzana en los tratamientos $\mathrm{T}_{2}$ y $\mathrm{T}_{3}$, permitió una mayor concentración de ácido ascórbico en comparación con las mermeladas que no se les adicionó manzana orgánica. Los resultados obtenidos aquí corroboran lo señalado por Gattás [18], quien menciona que la adición de un componente frutal extra, incrementa la ganancia nutritiva en la elaboración de un componente alimentario. La pérdida del contenido de ácido ascórbico es influenciada por el retiro de la piel de las frutas; el cual contiene el mayor contenido de ácido ascórbico; para el caso del arándano y la frambuesa, por causa del filtrado realizado y en el caso de la manzana, por el pelado de la fruta [18]. En este sentido, Fellows [19], menciona la perdida de Vitamina C, por un breve tiempo de ebullición de los alimentos y además, el proceso de congelado en la fruta, disminuye al menos un $30 \%$ la cantidad de ácido ascórbico [20].

Las mermeladas elaboradas con los tratamientos $\mathrm{T}_{0} \mathrm{y} \mathrm{T}_{2}$, no presentan diferencias significativas en la cantidad de azúcares totales, debido a la utilización del edulcorante no nutritivo (stevia). Tal y como lo destaca Brouns et al. [21], que al utilizar un edulcorante no nutritivo en su totalidad o en parte, disminuye la cantidad de azucares totales. La mermelada elaborada con el tratamiento $\mathrm{T}_{3}$, presentó menor cantidad de azúcares totales en comparación con el tratamiento $\mathrm{T}_{1}$, debido a que poseía un 50\% menos de azúcar granulada orgánica.

De acuerdo con los resultados obtenidos en las mermeladas elaboradas, los tratamientos $\mathrm{T}_{1}$ y $\mathrm{T}_{3}$, no presentaron diferencias significativas en el porcentaje de pectina entre ellos; sin embargo, la mermelada realizada con el tratamiento $\mathrm{T}_{0}$ es la que presentó la mayor concentración de pectato de calcio. Según Coronado y Hilario [22], la pectina es una sustancia natural que se encuentra en las células de las frutas, y cuya función, consiste en gelificar; es decir que, a mayor concentración de fruta, mayor es la cantidad de pectina, lo que coincide con la información de las mermeladas elaboradas en la presente investigación.

La mezcla de Stevia, azúcar, fruta orgánica y manzana orgánica $\left(\mathrm{T}_{3}\right)$, demostró ser el tratamiento con mayor sabor "dulce" y "agradable", percibido y comentado por los panelistas con valores entre 12.07 y 12.23 , lo que coincide con lo planteado por Rubio et al.[23], aunque mientras mayor sea la concentración de Stevia en el producto, se incrementará el "sabor amargo".

Las mermeladas elaboradas con los tratamientos $\mathrm{T}_{0} \mathrm{y}$ $\mathrm{T}_{2}$, no presentaron diferencias significativas en el color entre las muestras, según la percepción reflejada entre los panelistas; debido a que la Stevia, favorece el 
mantenimiento del color natural del producto [23]; sin embargo, las mermeladas elaboradas con los tratamientos $\mathrm{T}_{1}$ y $\mathrm{T}_{3}$, si presentan diferencias significativas entre ellos, lo que probablemente se debió a la aplicación de edulcorantes (azúcar) que se evidenció la afectación en el color del producto final; obteniendo una tonalidad más oscura, tal y como lo señala Raza et al. [24].

Todos los tratamientos en las mermeladas elaboradas, presentaron diferencias significativas en su textura. Destaca Boatella et al. [25] que la adición de azúcar y/o manzana, incrementa la textura de las mermeladas; lo que se demuestra en las mermeladas elaboradas con los tratamientos $\mathrm{T}_{1}, \mathrm{~T}_{2}$ y $_{3}$, los cuales presentan un incremento en la textura en comparación con el tratamiento $\mathrm{T}_{0}$.

Todas las mermeladas elaboradas, presentaron diferencias significativas en el aroma, en sus distintos tratamientos. Según Torregroza et al. [26], el remplazo total de un edulcorante nutritivo afecta en la mermelada modificando sus propiedades organolépticas. Al utilizar completamente la adición de stevia, no se evidenció incremento en el aroma frutal en la mermelada preparada, pero al utilizar la combinación de los 2 edulcorantes (azúcar y stevia), sí aumentó el aroma frutal; sin embargo la utilización de solo azúcar y fruta $\left(\mathrm{T}_{1}\right)$, produjo que el aroma frutal, se enmascarara con el aroma a caramelo.

Todos los tratamientos en las mermeladas elaboradas, presentaron diferencias significativas entre ellos, respecto a la aceptabilidad (figura 10), en donde la mermelada elaborada con el tratamiento $\mathrm{T}_{3}$, fue la que presentó los mayores promedios obtenidos; calificándolo con nota 8 "me gusta mucho". Los panelistas señalaron en sus observaciones, que la mermelada elaborada con el tratamiento $\mathrm{T}_{3}$ fue la que más les agradó, señalando que tenía un gusto "agradable" y no "hostigante". Sin embargo, también se destacó que el tratamiento $\mathrm{T}_{1}$ otorgó a las mermeladas, valores entre 7 y 7.2 calificados como "me gusta moderadamente" según la percepción de los panelistas.

Según Rocha et al. [27], mientras mayor es la concentración de Stevia, aumenta el sabor amargo en el producto final, lo que generaría disgusto en el consumidor. Al reducir la cantidad de Stevia utilizada en la presente investigación, se generó una mayor aceptación del producto final. Al contener una mezcla de azúcar y manzana orgánica, generó una buena concentración de pectina, lo que coincide con los hallazgos de Boatella et al. [25], lo que permitió que en la presente investigación, se obtuviera una mejor textura en la mermelada para el consumidor. La cantidad de contenido de ácido ascórbico (vitamina C), varió en cada elaboración de mermelada, en donde la mayor concentración, se obtuvo en los tratamientos en los cuales se adicionó un componente frutal extra a la mermelada, en el cual, el tratamiento $\mathrm{T}_{3}$ (fruta orgánica + manzana Granny Smith + stevia + azúcar orgánica), presento la mayor cantidad de ácido ascórbico en concentraciones de 24.5 y $25.5 \mathrm{mg}$ de ácido ascórbico. $100 \mathrm{~g}^{-1}$ de muestra.

\section{Conclusiones}

La elaboración de mermelada de arándano y frambuesa orgánicos, sellados al vacío, permitió la conservación de los valores nutricionales y favoreció los valores sensoriales durante 35 días.

La adición de stevia, no presentó diferencias significativas en la cantidad de azúcares totales entre las mermeladas elaboradas con el tratamiento $\mathrm{T}_{0}$ (fruta orgánica + conservantes) y $\mathrm{T}_{2}$ (fruta orgánica + stevia), por lo cual, la Stevia no incrementó significativamente los azúcares totales y los ${ }^{\circ}$ Brix en la mermelada elaborada.

El contenido de ácido cítrico y pectato de calcio (pectina), se ven afectados de acuerdo con la concentración de fruta en cada mermelada; donde existió una mayor concentración de fruta, hubo una mayor concentración de ácido cítrico y pectina; como se demostró en las mermeladas elaboradas con los tratamientos $\mathrm{T}_{0} \mathrm{y} \mathrm{T}_{2}$.

La mermelada elaborada con el tratamiento $\mathrm{T}_{2}$, presentó los valores más elevados en sabor y aroma, mientras que el tratamiento $T_{1}$, exhibió los valores más altos en textura y sabor, de acuerdo con la perceptibilidad de los panelistas.

La mermelada que presentó la mayor aceptabilidad, fue la elaborada con el tratamiento $\mathrm{T}_{3}$, de acuerdo a lo percibido por el grupo de panelistas, el producto fue clasificado con un promedio de 8 , lo cual pone de manifiesto que la mermelada elaborada con el mix 
de arándanos y frambuesas, genera satisfacción en el consumidor, y los panelistas la catalogaron con un alto nivel de aceptabilidad ("me gusta mucho").

\section{Consentimiento de publicación}

Los autores leyeron y aprobaron el manuscrito final.

\section{Conflicto de intereses}

Los autores declaran no tener conflictos de interés. Este documento solo refleja sus puntos de vista y no los de las instituciones a las que cada autor pertenece.

\section{Perfil de autoría}

\begin{abstract}
Nelson Eduardo Loyola López Ingeniero Agrónomo Pontificia Universidad Católica de Valparaíso, Chile; Licenciado en Agronomía Pontificia Universidad Católica de Valparaíso, Chile; Diploma Food Processing and Quality Assurance Wageningen University, Holanda; Master of Science Horticulture and Lanscape Architecture Washington State University, Estado de Washington, EE.UU. Asistente de investigación en proyecto de papas para agro industria en Departamento Horticultura, Washington State University, Estado de Washington, EE.UU.; Gerente empresa agro industria en el área de exportación. Director Departamento Ciencias Agrarias, Facultad Ciencias Agrarias y Forestales, Universidad Católica del Maule. Decano Facultad Ciencias Agrarias y Forestales. Universidad Católica del Maule, Chile. Investigador asociado en temas de agroindustria financiado por Fondo Innovación Agraria en la línea de producción orgánica. Numerosas publicaciones tanto Scielo, como ISI web of Science, nacionales e internacionales. Autor de dos libros de fundamentos hortofrutícolas; Autor de dos patentes en tramitación y referidas a alimentos procesados.
\end{abstract}

\section{Carlos Alberto Acuña Carrasco}

Químico laboratorista Universidad de Chile; ha realizado diversas actividades industriales, como las llevado a cabo en la Industria Azucarera Nacional Iansa Curico; Fideos y Alimentos Carozzi S.A.; Rocofrut Curico, planta de cerezas; Procefrut Curico, exportación alcachofas; kiwi; cerezas; Agroindustrial Surfrut S.A.; Prodasa división congelados y deshidratados; realiza actividades de docencia

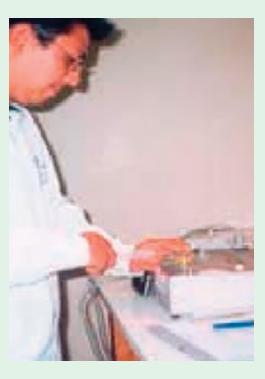
en el Instituto Profesional AIEP, en el programa Agroindustria y en la Universidad Católica del Maule. Imparte consultorías y asesorías especializada en temas se agroindustria a diversos sectores.

\section{Referencias}

[1] García Rubio J, García González G, Ciorda Ara M. Situación actual del cultivo del arándano en el mundo. Tecnología Agroalimentaria 2013:5-8. http://www.serida.org/pdfs/5566.pdf

[2] Sánchez Rodríguez G. La red de valor de la zarzamora. El cluster de Los Reyes, Michoacán un ejemplo de reconversión competitiva. Michiacán, México: 2008.

[3] Kim MJ, Sutton KL, Harris GK. Raspberries and Related Fruits. Encyclopedia of Food and Health Reference Module in Food Science 2016:586-91. https://doi.org/10.1016/B9780-12-384947-2.00586-9.

[4] González G, Aguilera F, D’Afonseca V. Transcriptome profiling of raspberry (Rubus idaeus Var. Amira) in response to infection by tomato ringspot virus (ToRSV). Heliyon 2020;6:e04518. https://doi.org/10.1016/J.HELIYON.2020. E04518.

[5] Codex Alimentarius. Normas Internacionales de los Alimentos. Norma para las confituras, jaleas y mermeladas CXS 296-2009. Adoptada en 2009. Enmendada en 2017, 2020. Roma, Italia: 2020. https://www.fao.org/fao-who-codexalimentarius/shproxy/en/?lnk=1\&url=https $\% 253 \mathrm{~A} \% 252 \mathrm{~F} \% 252 \mathrm{Fworkspace}$. fao.org $\% 252$ Fsites $\% 252$ Fcodex $\% 252$ FStandards $\% 252$ FCXS \%2B296-2009\%252FCXS_296s.pdf

[6] González Zagal C. Balance general de la industria de frambuesas congeladas. Santiago de Chile, Chile: 2014. https://www.odepa.gob.cl/wp-content/uploads/2014/02/ Frambuesas201402.pdf

[7] Food and Agriculture Organization of the United Nations (FAO). The future of food and agriculture. Trends and challenges. Rome, Italy: 2017. https://www.fao.org/3/i6583e/ i6583e.pdf

[8] Mettler Toledo. Balanzas y básculas AB-S \& PB-S. Folleto técnico. Greifensee, Suiza: 2014. https://www.mt.com/ dam/LabDiv/Campaigns/cosmetics/catalogs/METTLER_ TOLEDO_LabCat_2014_15_ES.pdf

[9] Official Methods of analysis of the Association of Official Analytical Chemists (AOAC). Official Methods of Analysis 932.12 Solid (Soluble) in Fruit Products. Refractometer Method. Arlington- Virginia, USA: 1990.

[10] Featherstone S. Jams, jellies, and related products. vol. 3. 3rd ed. Sawston, United Kingdom: Woodhead Publishing Books. Elsevier; 2016. https://doi.org/10.1016/B978-0-85709-679$1.00009-\mathrm{X}$.

[11] Shoji K, Schudel S, Onwude D, Shrivastava C, Defraeye T. Mapping the postharvest life of imported fruits from packhouse to retail stores using physics-based digital twins. 
Resources, Conservation and Recycling 2022;176:105914. https://doi.org/10.1016/J.RESCONREC.2021.105914.

[12] Association of Official Agricultural Chemists (AOAC). Official Methods of Analysis 967.21 Official titratable 2,6-dichloroindophenol method for determination of vitamin $\mathrm{C}$ in fruit juices analysis. Official Methods of analysis of the Association of Official Analytical Chemists. RockvilleMaryland, USA: 1990.

[13] Analysis of the Association of Official Analytical Chemists (AOAC). AOAC Official Method 923.02. Carbon dioxide (total) in baking powders. Gasometric determination. Section 25.1.02. Rockville-Maryland, USA: 1995.

[14] Official Methods of analysis of the Association of Official Analytical Chemists. Official Methods of Analysis 942.15 Acidity (Titratable) Of Fruits Products. Arlington-Virginia, USA: 1990.

[15] Kirk R, Sawyer R, Egan H. Composición y análisis de alimentos de Pearson. 2nd ed. México, D.F., México: Editoral CECSA; 1996.

[16] Organización de las Naciones Unidas para la Alimentación y la Agricultura (FAO). Procesamiento a pequeña escala de frutas y hortalizas amazónicas nativas e introducidas. Roma, Italia: 1997.

[17] Yadav AK, Singh SV. Osmotic dehydration of fruits and vegetables: a review. Journal of Food Science and Technology 2014;51. https://doi.org/10.1007/s13197-012-0659-2.

[18] Gattás Zaror V. Guía de la composición nutricional de los alimentos naturales de la industria y preparaciones chilenas habituales. 2nd ed. Santiago de Chile, Chile: 2011. https:// bibliotecadigital.uchile.cl/permalink/56UDC_INST/llitqr/ alma991000848579703936

[19] Fellows P. Tecnología y procesamiento de los alimentos. Principios y prácticas. 1st ed. Zaragoza, España: Editorial Acribia; 1994. https://www.editorialacribia.com/media/ acribia/files/pdfcatalog-158.pdf
[20] Ramos Alvarado Z, García Panduro L, Pinedo Panduro L, Souza N R. Evaluación de factores de procesamiento y conservación de pulpa de Myrciaria Dubia H.B.K. (CamuCamu) que reducen el contenido de vitamina c (ácido ascórbico). Alimentaria 2002;2:89-99.

[21] Brouns F, Bjorck I, Frayn KN, Gibbs AL, Lang V, Slama $\mathrm{G}$, et al. Glycaemic index methodology. Nutrition Research Reviews 2005; 18:145-71. https://doi.org/10.1079/ NRR2005100.

[22] Coronado Trinidad M, Hilario Rosales R. Elaboración de mermeladas. Procesamiento de alimentos para pequeñas y microempresas agroindustriales. 1st ed. Lima, Perú: Centro de Investigación, Educación y Desarrollo (CIED); 2001.

[23] Rubio-Arraez S, Capella JV, Castelló ML, Ortolá MD. Physicochemical characteristics of citrus jelly with noncariogenic and functional sweeteners. Journal of Food Science and Technology 2016;53:3642-50. https://doi.org/10.1007/ s13197-016-2319-4.

[24] Raza Mehboob Ansari A, Jahangir Mulla S, Jairam Pramod G. Review on artificial sweeteners used in formulation of sugar free syrups. International Journal of Advances in Pharmaceutics 2015;4:5-9. https://doi.org/10.7439/ijap.

[25] Boatella Riera J, Codony Salcedo R, López Alegret P. Química y Bioquímica de los alimentos II . 1st ed. Barcelona, España: Universitat Barcelona, España; 2004.

[26] Torregroza Espinosa AM, Gomezcaceres Pérez LD carmen, Rodríguez Manrique JA, López Martínez RJ. Optimizing acceptability of mango jam enriched with pectin from cacao husk (Theobroma cacao L.). DYNA 2019; 86:292-6. https:// doi.org/10.15446/dyna.v86n208.72972.

[27] Rocha IF de O, Bolini HMA. Passion fruit juice with different sweeteners: sensory profile by descriptive analysis and acceptance. Food Science \& Nutrition 2015;3. https://doi. org/10.1002/fsn3.195. 